TUIMP-TH-97/80

hep-ph/9703408

\title{
Nonperturbative Explanation of the Enhancement Factors in QCD Sum Rule for the $\rho$ Meson
}

\author{
Shu-Quan Nie \\ Department of Physics, Tsinghua University, Beijing 100084, China \\ Yu-Ping Kuang Qing Wang Yu-Ping Yi \\ China Center of Advanced Science and Technology (World Laboratory), \\ P. O. Box 8730, Beijing 100080, China \\ and \\ Institute of Modern Physics, Tsinghua University, Beijing 100084, Chind
}

\begin{abstract}
Taking the sum rule for the $\rho$ meson as an example, we study the possibility of explaining the phenomenological enhancement factors for certain terms in the vacuum expectation value of the operator product expansion in the QCD sum rule. We take a QCD motivated extended Nambu-Jona-Lasinio (ENJL) model as the low energy effective Lagrangian for QCD with which we calculate the nonperpturbative contributions to the vaccum condensate expansion to obtain the enhancement factors. Our result shows that such nonperturbative contrbutions can cause large enough enhancement factors which can be consistent with the phenomenological values.
\end{abstract}

PACS numbers: 11.55.Hx, 12.38.-t, 11.15.Pg

Typeset Using REVTEX

*Mailing address. 


\section{INTRODUCTION}

QCD sum rule [1] is a useful semi-phenomenological approach to hadron physics in which nonperturbative vacuum condensate (VC) effects are taken into account. The sum rule relates physical observables to certain VC's through the vacuum expectation values (VEV's) of products of current operators $\left\langle T j^{A}(x) j^{B}(0)\right\rangle$ with the vacuum condensate expansion (VCE)

$$
i \int d^{4} x e^{i q \cdot x}\left\langle T j^{A}(x) j^{B}(0)\right\rangle=F^{A B}(q) \sum_{n} C_{n}\left(Q^{2}\right)\left\langle O_{n}\right\rangle,
$$

where $F^{A B}(q)$ is a tensor factor characterizing the tensor structure in the indices $A$ and $B$, $Q^{2} \equiv-q^{2},\left\langle O_{n}\right\rangle$ 's are VC's of various dimensions. Usually, up to the VC's of dimension-6 operators (four-quark operators) are taken into account in (1). In most applications of the QCD sum rule, the following approaches are taken:

(i) as a basic assumption, the coefficients $C_{n}$ 's, at least in the first few terms, are assumed to be just the Wilson coefficients in the standard operator product expansion (OPE) determined merely from perturbative QCD (PQCD), and nonperturbative effects all reside in the VC's $\left\langle O_{n}\right\rangle$ 's [1];

(ii) $\left\langle O_{n}\right\rangle$ 's are treated as free parameters determined by experimental inputs, and to reduce the number of free parameters, people often make the simple factorization approximation to express the four-quark condensate $(4 \mathrm{QC})$ in terms of the two-quark condensate $(2 \mathrm{QC})$ in the following way [1].

$$
\left\langle\bar{\psi} \Gamma_{1} \psi \bar{\psi} \Gamma_{2} \psi\right\rangle \approx N^{-2}\left[\operatorname{Tr} \Gamma_{1} \operatorname{Tr} \Gamma_{2}-\operatorname{Tr}\left(\Gamma_{1} \Gamma_{2}\right)\right]\langle\bar{\psi} \psi\rangle^{2},
$$

where $\psi$ is the three-flavor light quark field, the Lorentz structure of $\Gamma_{1,2}$ is 1 for scalar (S), $i \gamma_{5}$ for pseudoscalar (P), $\gamma_{\mu}$ for vector $(\mathrm{V}), \gamma_{5} \gamma_{\mu}$ for axial-vector (A), $\sigma_{\mu \nu}$ for tensor (T) (for color and flavor non-singlet, the color and flavor group generators $\lambda_{\alpha} / 2, t_{i}$ should also be included ), and $N=36$ is a normalization constant [1]. 
However, a careful analysis of the QCD sum rule for the $\rho$ meson [2] shows that the theoretical results are not consistent with the experiments and phenomenologically the demension-6 4QC term should be enhanced by a factor

$$
\kappa_{4 Q} \approx 3-6.6
$$

for fitting the data [2]. Studies of other processes with the same approach such as the sum rules for baryons [3] and pseudoscalar meson [4] lead to similar conclusion that an enhancement factor around $\kappa_{4 Q} \approx 4$ is needed for fitting the data.

There is an alternative analysis from the finite energy sum rule which leads to similar but somewhat different results that the dimension-6 4QC term and the dimension-4 gluon condensate plus two-quark condensate terms (Actually, the gluon condensate term dominates.) should be enhanced by factors

$$
\kappa_{4 Q} \approx 5-8, \quad \kappa_{G} \approx 2-5
$$

respectively [5].

So far there is no successful theoretical explanation of the enhancement factors. In our previous paper [6], we kept assumption (i) and looked at the possibility of explaining the enhancement factor $\kappa_{4 Q}$ in (3) by taking account of the non-factorized parts of the 4QC's, i.e. modifying approximation (ii). We took the QCD motivated Nambu- Jona-Lasinio (NJL) model by Bijnens, Bruno, and de Rafael [7] as the low energy effective Lagrangian of QCD to calculate the non-factorized parts of the 4QC's by means of a nonperturbative method for the effective potential for local composite operators [8], and the obtained non-factorized parts of the 4QC's are of the next-to-the-leading order in the $1 / N_{c}$ expansion. Our conclusion is that for reasonable values of the parameters in the model, the obtained enhancement factor $\kappa_{4 Q}$ is only a few percent larger than unity, so that it is too small to account for (3). The next reasonable trial is to consider certain modification of assumption (i) since the $\rho$ meson lies below the chiral symmetry breaking scale wherein nonperturbative effects are important. Although an argument considering nonperturbative effects from instantons in 
the dilute gas approximation has been given in Ref. [1] expecting that assumption (i) may work up to about ten terms in (1), the analyses in Refs. [2] [5] show that the real situation is not so optimistic. In this paper, inspired by a recent paper by Yamawaki and Zakharov [9], we study the possibility of explaining the enhancement factors by taking into account certain nonperturbative contributions to the VCE in which nonperturbative effects not only reside in the condensates but also affect the coefficients in the VCE so that assumption (i) is modified. We take the whole extended Nambu- Jona-Lasinio (ENJL) Lagrangian in Ref. [7] as the low energy effective Lagrangian of the quark and gluon system, and calculate the nonperturbative contributions to the VCE from the NJL four-fermion interactions to the precision of the leading order of $1 / N_{c}$ expansion. Our results show that, taking only four-fermion interactions of the scalar and pseudoscalar types [7], such modification mainly affects the 4QC terms in the VCE by causing additional 4QC's with different tensor structures which leads to a fairly large contribution to $\kappa_{4 Q}$. For reasonable parameters in

the model, it is really possible to obtain a large enough $\kappa_{4 Q}$ consistent with (3). We shall also show that, when including certain vector and axial-vector four-fermion interactions in the ENJL Lagrangian [7], it is also possible to explain the enhancement factors (4). This means that for low energy processes, QCD sum rule may give better results if the original assumption (i) is modified by taking account of more nonperturbative QCD effects.

This paper is organized as follows. Sec.II is the theoretical aspect of this study. In Sec.III we present the calculations of the nonperturbative contributions to the VCE up to the 4QC terms from the ENJL model and the explanation of the enhancement factors. Conclusions are given in Sec.IV.

\section{THE THEORETICAL STRATEGY}

To study nonperturbative contributions, it is convenient to take a low energy effective Lagrangian of QCD including nonperturbative effects. So far there is no successful low energy effective Lagrangian derived from the first principles of QCD. The QCD motivated ENJL 
model by Bijnens, Bruno and de Rafael [7] contains reasonable QCD ingredient including chiral symmetry breaking, and can lead to rather successful phenomenological results [7]. So we take this model as the low energy effective Lagrangian for our calculations. The Lagrangian in the ENJL model in Ref. [7] is

$$
\mathcal{L}_{Q C D}=\mathcal{L}_{Q C D}^{\Lambda_{\chi}}+\mathcal{L}_{N J L}^{S P}+\mathcal{L}_{N J L}^{V A}
$$

where $\Lambda_{\chi}$ is a momentum cut-off below which the model serves as the low energy effective Lagrangian for $\mathrm{QCD}, \mathcal{L}_{Q C D}^{\Lambda_{\chi}}$ is of the same form of the $Q C D$ Lagrangian for momentum below $\Lambda_{\chi}$, and

$$
\begin{gathered}
\mathcal{L}_{N J L}^{S P}=\frac{8 \pi^{2} G_{S}}{N_{c} \Lambda_{\chi}^{2}} \sum_{a b}\left(\bar{\psi}_{R}^{a} \psi_{L}^{b}\right)\left(\bar{\psi}_{L}^{b} \psi_{R}^{a}\right) \\
=\frac{2 \pi^{2} G_{S}}{3 N_{c} \Lambda_{\chi}^{2}}\left[(\bar{\psi} \psi)^{2}+\left(\bar{\psi} i \gamma_{5} \psi\right)^{2}\right] \\
+\frac{4 \pi^{2} G_{S}}{N_{c} \Lambda_{\chi}^{2}} \sum_{i=1}^{8}\left[\left(\bar{\psi} t_{i} \psi\right)^{2}+\left(\bar{\psi} i \gamma_{5} t_{i} \psi\right)^{2}\right], \\
\mathcal{L}_{N J L}^{V A}=-\frac{8 \pi^{2} G_{V}}{N_{c} \Lambda_{\chi}^{2}} \sum_{a b}\left[\left(\bar{\psi}_{L}^{a} \gamma^{\mu} \psi_{L}^{b}\right)\left(\bar{\psi}_{L}^{b} \gamma_{\mu} \psi_{L}^{a}\right)+\left(\bar{\psi}_{R}^{a} \gamma^{\mu} \psi_{R}^{b}\right)\left(\bar{\psi}_{R}^{b} \gamma_{\mu} \psi_{R}^{a}\right)\right] \\
=-\frac{4 \pi^{2} G_{V}}{3 N_{c} \Lambda_{\chi}^{2}}\left[\left(\bar{\psi} \gamma^{\mu} \psi\right)^{2}+\left(\bar{\psi} \gamma_{5} \gamma^{\mu} \psi\right)^{2}\right] \\
-\frac{8 \pi^{2} G_{V}}{N_{c} \Lambda_{\chi}^{2}} \sum_{i=1}^{8}\left[\left(\bar{\psi} \gamma^{\mu} t_{i} \psi\right)^{2}+\left(\bar{\psi} \gamma_{5} \gamma^{\mu} t_{i} \psi\right)^{2}\right]
\end{gathered}
$$

in which $G_{S}$ and $G_{V}$ are two coupling constants treated as free parameters, and the flavor group generator $t_{i}$ is normalized as $\operatorname{tr}\left(t_{i} t_{j}\right)=\frac{1}{2} \delta_{i j}$. It is argued in Ref. [7] that the NJL type Lagrangian $\mathcal{L}_{N J L}^{S P}+\mathcal{L}_{N J L}^{V A}$ may be understood as coming from integrating out the high momentum modes of quarks and gluons in the fundamental theory of QCD (However, $G_{S}$ and $G_{V}$ are taken as two independent free parameters here.) and is regarded as the part of $\mathcal{L}_{Q C D}$ responsible for chiral symmetry breaking, and $\mathcal{L}_{Q C D}^{\Lambda_{\chi}}$ provides perturbative corrections to the broken chiral symmetry state. This supports the idea of the chiral quark model 10 .

Our strategy is that we apply the Lagrangian (5)-(7) to energy scale lower than $\Lambda_{\chi}$, and take the ordinary PQCD approach for the energy range higher than $\Lambda_{\chi}$ where nonperturbative effects are not important. Since the structure of $\mathcal{L}_{Q C D}^{\Lambda_{\chi}}$ is the same as the original QCD 
Lagrangian, the pure perturbation results in the present appraoch are the same as those from the ordinary PQCD. The NJL Lagrangian $\mathcal{L}_{N J L}^{S P}+\mathcal{L}_{N J L}^{V A}$ not only gives rise to chiral symmetry breaking, but also give nontrivial contributions to the VCE which is what we are going to calculate.

There are three independent free parameters $G_{S}, G_{V}$, and $\Lambda_{\chi}$ in the ENJL model in Ref. [7]. They are related to the dynamical quark mass $M_{Q}$, the quark axial-vector coupling constant $g_{A}$, and the vector meson mass $M_{\rho}$ by the following relations [7]

$$
\begin{gathered}
1 / G_{S}=\left(M_{Q} / \Lambda_{\chi}\right)^{2} \Gamma\left(-1,\left(M_{Q} / \Lambda_{\chi}\right)^{2}\right)\left(1+\gamma_{-1}\right), \\
g_{A}=\frac{1}{1+4 G_{V}\left(M_{Q} / \Lambda_{\chi}\right)^{2} \Gamma\left(0,\left(M_{Q} / \Lambda_{\chi}\right)^{2}\right)\left(1+\gamma_{01}\right)}, \\
\Lambda_{\chi}^{2}=\frac{2}{3} M_{\rho}^{2} G_{V} \Gamma\left(0,\left(M_{Q} / \Lambda_{\chi}\right)^{2}\right)\left(1+\gamma_{03}\right),
\end{gathered}
$$

where $\Gamma\left(n-2,\left(M_{Q} / \Lambda_{\chi}\right)^{2}\right)$ is the incomplete gamma function [7], and $\gamma_{-1}, \gamma_{01}$, and $\gamma_{03}$ are perturbative corrections. Actually $M_{Q}, \Lambda_{\chi}$, and $g_{A}$ are taken as the input parameters to fit the data in Ref. [7]. In this paper, we calculate the contributions of the NJL Lagrangian $\mathcal{L}_{N J L}^{S P}+\mathcal{L}_{N J L}^{V A}$ to the precision of the leading order in the $1 / N_{c^{-}}$expansion, i.e. the uncertainty of the calculation is of the order of $30 \%$. With this uncertianty, we shall neglect the small quatities $\gamma_{-1}, \gamma_{01}$ and $\gamma_{03}$ throughout this paper. Moreover, we shall also neglect the non-factorized parts of the 4QC's given in Ref. [6] since they are of higher order in the expansion.

In calculating Feynman diagrams to the precision of the leading term in $1 / N_{c}$-expansion, we should sum up all open-end chain diagrams attached to each quark line (cf. Fig.1). Note that only the flavor-singlet scalar four- fermion interaction contributes to these chain diagrams. It is easy to see that the effect of summing up such chain diagrams attached to a quark line is just providing a dynamical mass term $M_{Q}$ (arising from $\langle\bar{\psi} \psi\rangle \neq 0$ ) added to the current quark mass $m$ to form the full quark mass of this quark line. The relation 
Gs

:

Gs 
Fig. 1. Chain diagrams in the calculation of the leading contribution in $1 / N_{c}$ expansion.

between $M_{Q}$ and $\langle\bar{\psi} \psi\rangle$ can be obtained as follows. In the large- $N_{c}$ limit, the gap equation is [7]

$$
\langle\bar{\psi} \psi\rangle=-\frac{3 N_{c}}{4 \pi^{2}} M_{Q}^{3} \Gamma\left(-1,\left(M_{Q}^{2} / \Lambda_{\chi}^{2}\right)\right)
$$

in which the chiral limit relation $\langle\bar{u} u\rangle=\langle\bar{d} d\rangle=\langle\bar{s} s\rangle=\frac{1}{3}\langle\bar{\psi} \psi\rangle$ is used. the function $\Gamma\left(-1,\left(M_{Q}^{2} / \Lambda_{\chi}^{2}\right)\right)$ can be eliminated by using (8). neglecting $\gamma_{-1}$ in (8), we get from (11) and (8)

$$
M_{Q}=-\frac{4 \pi^{2} G_{S}}{3 N_{c} \Lambda_{\chi}^{2}}\langle\bar{\psi} \psi\rangle .
$$

Hence $M_{Q}$ is proportional to $\langle\bar{\psi} \psi\rangle$. Since QCD sum rule concerns the VCE in which various dimensional quark condensates are put as explicit terms, all the effects of $M_{Q}$ should be put into the explicit quark condensates in the VCE by definition. Considering these nonperturbative effects, (1) becomes

$$
i \int d^{4} x e^{i q \cdot x}\left\langle j^{A}(x) j^{B}(0)\right\rangle=F^{A B}(q) \sum_{n} \tilde{C}_{n}\left(Q^{2}\right)\left\langle O_{n}\right\rangle
$$

in which the new coefficient $\tilde{C}_{n}\left(Q^{2}\right)$ in the resulting VCE is not the same as the original Wilson coefficient $C_{n}\left(Q^{2}\right)$ in the standard OPE. $\tilde{C}_{n}\left(Q^{2}\right)$ contains nonperturbative effects from the NJL Lagrangian. Generally, $\tilde{C}_{n}\left(Q^{2}\right)$ is a function of $\Lambda_{\chi}, G_{S}, G_{V}$, and the current quark mass $m$. In the theory with the Lagrangian (5)-(7), it is (13) rather than (1) that really matters in the QCD sum rule. Note that when calculating $\tilde{C}_{n}\left(Q^{2}\right)$, the quark mass in every quark line in the Feynman diagrams should be understood as the current quark mass $m$. Since the current quark mass $m$ is much smaller than $Q^{2} \sim M_{\rho}^{2}$, we treat $m^{2} / Q^{2}$ as a small perturbation in the following calculations. 


\section{CALCULATION OF $\tilde{\mathrm{C}}_{\mathbf{n}}$ 's AND THE EXPLANATION OF $\kappa$ 's}

In the sum rule for the $\rho$ meson, the currents $j^{A}, j^{B}$ are taken to be vector currents

$$
j_{\mu}=\frac{1}{2}\left(\bar{u} \gamma_{\mu} u-\bar{d} \gamma_{\nu} d\right)=\bar{\psi} t_{3} \psi,
$$

where $t_{3}$ is the flavor group generator in (6)-(7). The VCE of the product of the currents takes the form

$$
\begin{aligned}
& i \int d x e^{i q x}\left\langle 0\left|T j_{\mu}(x) j_{\nu}(0)\right| 0\right\rangle=\left(q_{\mu} q_{\nu}-q^{2} g_{\mu \nu}\right) \Pi\left(q^{2}\right), \\
& \Pi\left(Q^{2}\right)=\tilde{C}_{I}\langle I\rangle+\tilde{C}_{2 Q}\left\langle\bar{\psi} M_{u, d} \psi\right\rangle+\tilde{C}_{G}\left\langle G_{\mu \nu}^{a} G^{a \mu \nu}\right\rangle+\tilde{C}_{4 Q} \sum_{\Gamma}\langle\bar{\psi} \Gamma \psi \bar{\psi} \Gamma \psi\rangle+\cdots,
\end{aligned}
$$

where $M_{u, d}$ is the $u-, d$-quark mass matrix. We are going to calculate the four terms on the right-hand-side of (15) including the nonperturbative contributions from the NJL Lagrangian in (5)-(7).

\section{Model with $G_{S} \neq 0, G_{V}=0$.}

There are five different ways of fitting the data presented in Ref. [7], which determine different sets of values of $M_{Q}, \Lambda_{\chi}$, and $g_{A}$, and the predictions are all successful. The simplest one of the fits is their Fit 4 in which $g_{A}=1$. From (9) we see that this corresponds to $G_{V}=0$ which makes the calculation of the nonperturbative contributions easiest. Moreover, it is explained by Weinberg [11] that, in the large $N_{c}$ limit, $g_{A}$ should actually be unity in the constituent quark model. In view of this, we take the set Fit 4 in Ref. [7] in this subsection, and by means of (8)-(10), it leads to $G_{S}=1.19, G_{V}=0, \Lambda_{\chi}=667$ $\mathrm{MeV}$. Since the uncertainty in our calculation is of the order of 30\%, we are not taking these 


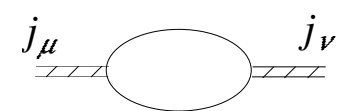

(a)

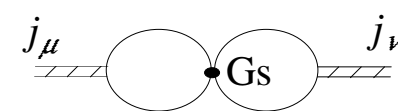

(b) 
Fig. 2. Example of Feynman diagrams for leading contributions to

$$
\tilde{C}_{I}\langle I\rangle \text {. (a) PQCD contribution; (b) } \mathcal{L}_{N J L}^{S P} \text { contribution. }
$$

precise values seriously and, instead, we shall consider a reasonable range of the parameters.

Now we calculate the coefficients in (15). Examples of the Feynman diagram of the leading contribution of PQCD and $\mathcal{L}_{N J L}^{S P}$ to $\tilde{C}_{I}\langle I\rangle$ is shown in Fig.2. This has been studied in Ref. [9]. The first nonvanishing contribution comes from the term suppressed by $G_{S} m^{2} / Q^{2}$ which is negligibly small compared with our theoretical uncertainty. Thus In the present appraoch $\tilde{C}_{I}\left(Q^{2}\right)$ is fairly well given by the standard Wilson coefficient $C_{I}\left(Q^{2}\right)$ from $P Q C D$ given in Ref. [1], i.e.

$$
\tilde{C}_{I} \approx C_{I}=-\frac{1}{8 \pi^{2}} \ln \frac{Q^{2}}{\mu^{2}} .
$$

The PQCD result of the $\tilde{C}_{2 Q}\langle\bar{\psi} M \psi\rangle$ term is proportional to the current quark mass $m$ [1] [cf. Fig.3(a)]. Examples of the Feynman diagram for the $\mathcal{L}_{N J L}^{S P}$ contribution to the $\tilde{C}_{2 Q}\langle\bar{\psi} M \psi\rangle$ term is shown in Fig.3(b) (This corresponds to the effect linear in $M_{Q}$. ). It is easy to see that the first nonvanishing contribution is of the order of $m \frac{m^{2}}{Q^{2}}$ and is thus also negligible relative to the PQCD contribution to the present precision. Hence $\tilde{C}_{2 Q}\left(Q^{2}\right)$ in the present approach is also mainly given by the standard Wilson coefficient $C_{2 Q}\left(Q^{2}\right)$ from $P Q C D$ given in Ref. [1], i.e.

$$
\tilde{C}_{2 Q}\left\langle\bar{\psi} M_{u, d} \psi\right\rangle \approx C_{2 Q}\left\langle\bar{\psi} M_{u, d} \psi\right\rangle=\frac{1}{2 Q^{4}}\left(m_{u}\langle\bar{u} u\rangle+m_{d}\langle\bar{d} d\rangle\right)
$$


13 


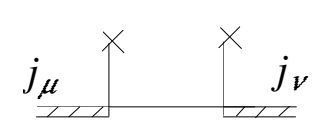

(a)

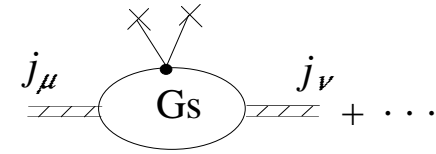

(b) 
Fig. 3. Example of Feynman diagrams for leading contributions to $\tilde{C}_{2 Q}\left\langle\bar{\psi} M_{u d} \psi\right\rangle$. (a) PQCD contribution; (b) $\mathcal{L}_{N J L}^{S P}$ contribution.

The situation of the $\tilde{C}_{G}\left\langle G_{\mu \nu}^{a} G^{a \mu \nu}\right\rangle$ is similar. Examples of the Feynman diagrams for leading PQCD and $\mathcal{L}_{N J L}^{S P}$ contributions to this term are shown in Fig.4. The first nonvanishing contribution in Fig.4(b) is also of order $G_{S} m^{2} / Q^{2}$ which highly suppresses the nonperturbative contribution. Thus $\tilde{C}_{G}\left(Q^{2}\right)$ is also maily given by the standard Wilson coefficient $C_{G}\left(Q^{2}\right)$ from $P Q C D$ given in Ref. [1], i.e.

$$
\tilde{C}_{G}\left\langle G_{\mu \nu}^{a} G^{a \mu \nu}\right\rangle \approx C_{G}\left\langle G_{\mu \nu}^{a} G^{a \mu \nu}\right\rangle=\frac{\alpha_{s}}{24 \pi Q^{4}}\left\langle G_{\mu \nu}^{a} G^{a \mu \nu}\right\rangle
$$

For the 4QC term, the situation is completely different. Examples of the Feynman diagrams for leading order PQCD and $\mathcal{L}_{N J L}^{S P}$ contributions are shown in Fig.5. Unlike the previous cases (cf. Figs.2(b), 3(b), 4(b), Fig.5(b) is of tree-level rather than loop-level, therefore this contribution is large. The leading PQCD [Fig.5(a)] formula given in Ref. [1] can be written as

$$
\begin{aligned}
& \left.\sum_{\Gamma} \tilde{C}_{4 Q}^{\Gamma}\langle\bar{\psi} \Gamma \psi \bar{\psi} \Gamma \psi\rangle\right|_{P Q C D}=\sum_{\Gamma} C_{4 Q}^{\Gamma}\langle\bar{\psi} \Gamma \psi \bar{\psi} \Gamma \psi\rangle \\
& =-\frac{2 \pi \alpha_{s}}{Q^{6}}\left[\left\langle\left(\bar{\psi} \gamma_{\mu} \gamma_{5} \lambda^{a} t_{3} \psi\right)^{2}\right\rangle+\frac{1}{9}\left\langle\bar{\psi} \gamma_{\mu} \lambda^{a}\left(\frac{1}{3}+\frac{1}{\sqrt{3}} t_{8}\right) \psi \bar{\psi} \gamma_{\mu} \lambda^{a} \psi\right\rangle\right]
\end{aligned}
$$

where $C_{4 Q}^{\Gamma}\left(Q^{2}\right)$ is the standard Wilson coefficient. Note that there are only VEV's of products of vector and axial-vector currents. The calculation of Fig.5(b) is straightforward but lengthy. There is also contribution to the 4QC term from the expansion of $\langle\bar{\psi}(x) \psi(0)\rangle$ arising in Fig.3(b). Adding the two contributions together, we get 


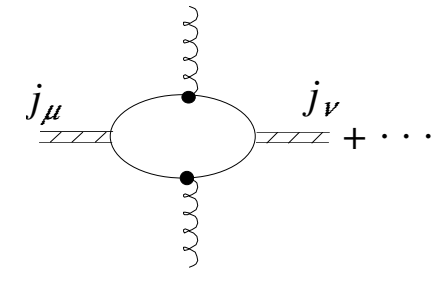

(a)

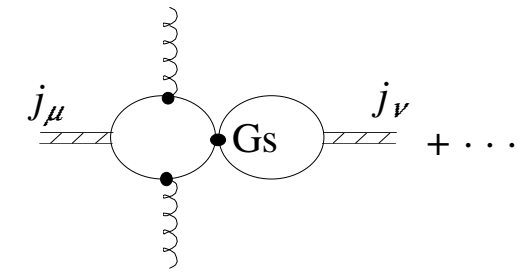

(b) 
Fig. 4. Example of Feynman diagrams for leading contributions to $\tilde{C}_{G}\left\langle G_{\mu \nu}^{a} G^{a \mu \nu}\right\rangle$. (a) PQCD

$$
\begin{gathered}
\text { contribution; (b) } \mathcal{L}_{N J L}^{S P} \text { contribution. } \\
\left.\sum_{\Gamma} \tilde{C}_{4 Q}^{\Gamma}\langle\bar{\psi} \gamma \psi \bar{\psi} \Gamma \psi\rangle\right|_{N J L}=-\frac{2 \pi^{2} G_{S}}{N_{c} \Lambda_{\chi}^{2} Q^{4}}\left[\left\langle\left(\bar{\psi}\left(\frac{1}{3}+\frac{1}{\sqrt{3}}+t_{3}\right) \psi\right)^{2}\right\rangle+\left\langle\left(\bar{\psi}\left(\frac{1}{3}+\frac{1}{\sqrt{3}}-t_{3}\right) \psi\right)^{2}\right\rangle\right. \\
-\left\langle\bar{\psi} t_{+} \psi \bar{\psi} t_{-} \psi\right\rangle-\left\langle\bar{\psi} t_{-} \psi \bar{\psi} t_{+} \psi\right\rangle-\left\langle\left(\bar{\psi} \gamma_{5}\left(\frac{1}{3}+\frac{1}{\sqrt{3}}+t_{3}\right) \psi\right)^{2}\right\rangle-\left\langle\left(\bar{\psi} \gamma_{5}\left(\frac{1}{3}+\frac{1}{\sqrt{3}}-t_{3}\right) \psi\right)^{2}\right\rangle \\
\left.+\left\langle\bar{\psi} \gamma_{5} t_{+} \psi \bar{\psi} \gamma_{5} t_{-} \psi\right\rangle+\left\langle\bar{\psi} \gamma_{5} t_{-} \psi \bar{\psi} \gamma_{5} t_{+} \psi\right\rangle\right]
\end{gathered}
$$

where $t_{ \pm} \equiv t_{1} \pm i t_{2}$. We see that (20) contains the VEV's of products of scalar and pseudoscalar 4QC terms which are not suppressed by the current quark mass $m$, and thus are not small corrections to (19). Adding (19) and (20) together, we get the total 4QC term

$$
\sum_{\Gamma} \tilde{C}_{4 Q}^{\Gamma}\langle\bar{\psi} \Gamma \psi \bar{\psi} \Gamma \psi\rangle=\left.\sum_{\Gamma} \tilde{C}_{4 Q}^{\Gamma}\langle\bar{\psi} \Gamma \psi \bar{\psi} \Gamma \psi\rangle\right|_{P Q C D}+\left.\sum_{\Gamma} \tilde{C}_{4 Q}^{\Gamma}\langle\bar{\psi} \Gamma \psi \bar{\psi} \Gamma \psi\rangle\right|_{N J L}
$$

Compared with the standard OPE formula (19) used in Ref. [2], (21) contains extra significant terms which enhance the $4 Q C$ term in the VCE (15).

The above results are consistent with the analysis in Ref. [2], i.e. the first three terms in (15) are as usual, while the 4QC term is enhanced. With (21), we can define the enhancement factor $\kappa_{4 Q}$ in this approach as

$$
\kappa_{4 Q}=\frac{\sum_{\Gamma} \tilde{C}_{4 Q}^{\Gamma}\langle\bar{\psi} \Gamma \psi \bar{\psi} \Gamma \psi\rangle}{\sum_{\Gamma} C_{4 Q}^{\Gamma}\langle\bar{\psi} \Gamma \psi \bar{\psi} \Gamma \psi\rangle},
$$

where the numerator is given by (21) and the denominator is given by (19). The 4QC's in (19) and (21) contain their factorized parts given by (2) and non-factorized parts given in 


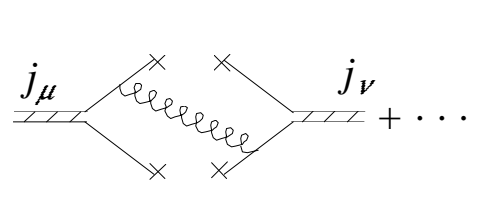

(a)

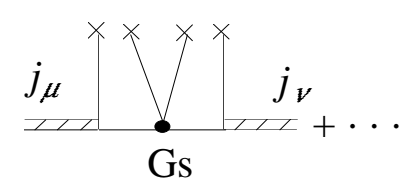

(b) 
Fig .5 Example of Feynman diagrams for leading contributions to $\sum_{\Gamma} \tilde{C}_{4 Q}^{\Gamma}\langle\bar{\psi} \Gamma \psi \bar{\psi} \Gamma \psi\rangle$

. (a) PQCD contribution; (b) $\mathcal{L}_{N J L}^{S P}$ contribution.

Ref. [6]. As we have stated, to the present precision we neglect the non-factorized parts. The factorized part of (19) reads [2]

$$
\left.\sum_{\Gamma} \tilde{C}_{4 Q}^{\Gamma}\langle\bar{\psi} \Gamma \psi \bar{\psi} \Gamma \psi\rangle\right|_{\substack{P Q C D \\ \text { factorized }}}=-\frac{14 \pi \alpha_{s}}{81}\left(1-\frac{1}{N_{c}^{2}}\right)\langle\bar{\psi} \psi\rangle^{2} .
$$

The factorized part of (20) obtained from (2) is

$$
\left.\sum_{\Gamma} \tilde{C}_{4 Q}^{\Gamma}\langle\bar{\psi} \Gamma \psi \bar{\psi} \Gamma \psi\rangle\right|_{\substack{N J L \\ \text { factorized }}}=-\frac{4 \pi^{2} G_{S}}{9 N_{c} \Lambda_{\chi}^{2} Q^{4}}\langle\bar{\psi} \psi\rangle^{2}
$$

From (12) we see that these factorized parts correspond to $M_{Q}^{2}$ contributions to the $4 \mathrm{QC}$ term. The explicit formula for $\kappa_{4 Q}$ for a given $Q^{2}$ in the present approach is

$$
\kappa_{4 Q} \approx 1+\frac{18 \pi N_{c} G_{S} Q^{2}}{7\left(N_{c}^{2}-1\right) \alpha_{s} \Lambda_{\chi}^{2}}
$$

We now estimate the value of $\kappa_{4 Q}$ in (25). We take $N_{c}=3$ and the PQCD coupling constant $\alpha_{s}\left(M_{\rho}\right) \approx 0.48$ which is obtained from the two-loop evolution formula from the world average value $\alpha_{s}\left(M_{Z}\right)=0.118$ [12]. From (8) we see that $G_{S}$ and $M_{Q}^{2} / \Lambda_{\chi}^{2}$ are related. We take $M_{Q}=199 \mathrm{MeV}$ from Fit 4 in Ref. [7], and take $\Lambda_{\chi}$ around the chiral symmetry breaking scale $\Lambda_{\chi} \sim 4 \pi f_{\pi} \sim 1.2 \mathrm{GeV}$. The values of $\kappa_{4 Q}$ with $Q^{2}=M_{\rho}^{2}$ for various values of $\Lambda_{\chi}$ are shown in Table 1. 
Table 1 Values of $\kappa_{4 Q}$ with $Q^{2}=M_{\rho}^{2}$ for various given values of $\Lambda_{\chi}$ in the

$$
G_{S} \neq 0, G_{V}=0 \text { model. }
$$

\begin{tabular}{ccc}
\hline \hline$\Lambda_{\chi}(\mathrm{GeV})$ & $G_{S}$ & $\kappa_{4 Q}$ \\
\hline 1.25 & 1.10 & 3.7 \\
1.10 & 1.15 & 4.7 \\
0.90 & 1.20 & 6.7 \\
0.80 & 1.25 & 8.3 \\
\hline \hline
\end{tabular}

We see that, with the uncertainty of the present calculation, the values of $\kappa_{4 Q}$ are consistent with the values in (3). Actually in the analysis in Ref. [2], Borel transformation

$$
\lim _{\substack{Q^{2} \rightarrow \infty, n \rightarrow \infty \\ Q^{2} / n \rightarrow M^{2}}} \frac{1}{(n-1) !}\left(Q^{2}\right)^{n}\left(-\frac{d}{d Q^{2}}\right)^{n}
$$

was applied. We have also looked at the result after applying the Borel transformation (26) with $M \approx M_{\rho}$. The results of $\kappa_{4 Q}$ is larger than those listed in Table 1. For example, for $\Lambda_{\chi}=1.25 \mathrm{GeV}$ and $1.10 \mathrm{GeV}$, we have $\kappa_{4 Q}=6.5$ and 8.3, respectively. These are still consistent with the phenomenological value in (3) considering the uncertainty of the present calculation. Thus the present theory with $G_{S} \neq 0$ and $G_{V}=0$ is compatible with the analysis in Ref. [2] that the first three terms in the VCE (15) are the same as those based on the basic assumption (i), while the $4 Q C$ term should be enhanced by a factor $\kappa_{4 Q}$ given in $(3)$.

\section{Model with $G_{S} \neq 0, G_{V} \neq 0$.}

The above model with $G_{S} \neq 0, G_{V}=0$ is not compatible with the enhancement factors (4) given in Ref. [5] in which $\kappa_{G}$ is not unity. Since the $\mathcal{L}_{N J L}^{V A}$ interactions give significant contributions to the first three terms in (15), it is interesting to see if a model with $G_{S} \neq 0$ and $G_{V} \neq 0$ can be compatible with (4).

We first consider the $\mathcal{L}_{N J L}^{V A}$ contributions to the 4QC term. The Feynman diagam for the leading contribution is the same as Fig.5(b) with $\mathcal{L}_{N J L}^{S P}$ replaced by $\mathcal{L}_{N J L}^{V A}$. After straight- 
forward calculations, we find that the factorized parts of $\left\langle\left(\bar{\psi} \gamma_{\mu} t_{3} \psi\right)^{2}\right\rangle$ and $\left\langle\left(\bar{\psi} \gamma_{\mu} \gamma_{5} t_{3} \psi\right)^{2}\right\rangle$ cancel each other so that the net contribution of the $\mathcal{L}_{N J L}^{V A}$ interaction to the $4 \mathrm{QC}$ term is just the corrections from the non-factorized parts which is negligible. So that the value of $\kappa_{4 Q}$ is not significantly affected by $G_{V} \neq 0$. Let us take Fit 1 in Ref. [7] as an example in which $g_{A}=0.61, \Lambda_{\chi}=1.16 \mathrm{GeV}, M_{Q}=265 \mathrm{MeV}\left(\gamma_{-1}=\gamma_{01}=\gamma_{03}=0\right.$ in Fit 1). Fixing this $M_{Q}$, the values of $G_{S}$ and $\kappa_{4 Q}$ with $Q^{2}=M_{\rho}^{2}$ for various values of $\Lambda_{\chi}$ are listed in Table 2. We see that these values are consistent with (4) considering the uncertainty of the present approach.

Table 2 Values of $\kappa_{4 Q}$ and $\kappa_{G}$ with $Q^{2}=M_{\rho}^{2}$ for various given values of $\Lambda_{\chi}$ in the $G_{S} \neq 0, G_{V} \neq 0$ model.

\begin{tabular}{ccccc}
\hline \hline$\Lambda_{\chi}(\mathrm{GeV})$ & $G_{S}$ & $G_{V}$ & $\kappa_{4 Q}$ & $\kappa_{G}$ \\
\hline 1.16 & 1.22 & 1.26 & 4.4 & 2.5 \\
1.00 & 1.28 & 1.06 & 5.8 & 2.7 \\
0.90 & 1.40 & 0.94 & 7.5 & 2.9 \\
0.80 & 1.42 & 0.84 & 9.3 & 3.1 \\
\hline \hline
\end{tabular}

We then look at the $\mathcal{L}_{N J L}^{V A}$ contributions to the gluon condensate term (the two-quark condensate term is relatively much smaller). The Feynman diagram is still Fig.4(b) with $\mathcal{L}_{N J L}^{S P}$ replaced by $\mathcal{L}_{N J L}^{V A}$. It is easy to see that only the $\left(\bar{\psi} \gamma_{\mu} t_{3} \psi\right)^{2}$ term in $(7)$ contributes. This gives rise to

$$
\tilde{C}_{G}\left(Q^{2}\right)=\left.\tilde{C}_{G}\left(Q^{2}\right)\right|_{P Q C D}+\left.\tilde{C}_{G}\left(Q^{2}\right)\right|_{N J L} \approx\left[1+\frac{4 G_{V} Q^{2}}{N_{c} \Lambda_{\chi}^{2}} \ln \frac{Q^{2}}{\mu^{2}}\right] C_{G}\left(Q^{2}\right) .
$$

Thus we have the enhancement factor

$$
\kappa_{G} \equiv \frac{\tilde{C}_{G}\left(Q^{2}\right)}{C_{G}\left(Q^{2}\right)} \approx 1+\frac{4 G_{V} Q^{2}}{N_{c} \Lambda_{\chi}^{2}} \ln \frac{Q^{2}}{\mu^{2}} .
$$

In the QCD sum rule, the scale $\mu$ is supposed to be the inverse of the confinement radius, $R_{\text {conf }}^{-1}$ [1]. Thus $\mu \approx \Lambda_{\overline{M S}} \approx 280 \mathrm{MeV}$. The values of $G_{V}$ and $\kappa_{G}$ with $Q^{2}=M_{\rho}^{2}$ for 
various values of $\Lambda_{\chi}$ are listed in Table 2. We see that these are also consistent with the $\kappa_{G}$ in (4).

The $\mathcal{L}_{N J L}^{V A}$ interactions will also give rise to the same enhancement factor as $\kappa_{G}$ for the $\tilde{C}_{I}\langle I\rangle$ term [9]. For $Q^{2} \approx M_{\rho}^{2}>\mu^{2}$, the PQCD value of $C_{I}$ [cf. (16)] is negative. Therefore this nonperturbative contribution makes $\tilde{C}_{I}$ more negative. We expect a global analysis in the spirit of Ref. [5] including the $\tilde{C}_{I}\langle I\rangle$ term and test the present prediction.

\section{CONCLUSIONS}

In this paper, taking the $\rho$-meson sum rule as an example, we have tried to modify the basic assumption of the QCD sum rule that the coefficients $C_{n}\left(Q^{2}\right)$ 's in (1) are merely determined by PQCD, and studied the possibility of explaining the phenomenological enhancement factors for certain terms in (1) [2] [5] by taking account of nonperturbative contributions to the VCE which lead to (15). We take the QCD motivated ENJL model in Ref. [7] as the low energy effective Lagrangian for QCD, and have calculated the nonperturbative contributions from the NJL Lagrangian to obtain the new coefficients $\tilde{C}_{n}$ 's to the precision of the leading order in the $1 / N_{c}$ expansion.

It is interesting that taking Fit 4 of Ref. [7], $\left(G_{S} \neq 0, G_{V}=0\right)$, our result shows that the first three terms in (15) are not affected much by the nonperturbative interactions, while the 4QC term contains extra terms with new tensor structures coming from the nonperturbative interactions and they are large compared with the PQCD result. Regarding these extra contributions as the theoretical source of the enhancement factor $\kappa_{4 Q}$, the obtained $\kappa_{4 Q}$ (cf. Table 1) is consistent with the phenomenological value (3), considering the theoretical uncertainty in the present calculation, for reasonable range of the parameters in the ENJL model.

Taking Fit 1 of Ref. [7], i.e. $G_{S} \neq 0, G_{V} \neq 0$, we have got another result that the obtained $\kappa_{4 Q}$ and $\kappa_{G}$ listed in Table 2 are all consistent with the results (4) given in Ref. [5]. However, in this model, $\tilde{C}_{I}\langle I\rangle$ will also be larger than $C_{I}\langle I\rangle$ by the same factor 
as $\kappa_{G}$ listed in Table 2, but this has not been discussed in Ref. [5]. We expect a further analysis of the $\tilde{C}_{I}\langle I\rangle$ term in the spirit of Ref. [5] to test whether the present prediction by Fit 1 is really right.

We conclude that it is possible to explain the enhancement factors in both Ref. [2] and [5] by taking different sets of parameters in the ENJL model in Ref. [7]. Within the 30\% uncertainty of the present calculation, the model with $G_{S} \neq 0, G_{V}=0$ can give a reasonable explanation of the analysis in Ref. [2] with the value of $\kappa_{4 Q}$ given in (3), and the model with $G_{S} \neq 0, G_{V} \neq 0$ ENJL model [0] can possibly explain the enhancement factors (4) given in Ref. [5]. In the present approach, the physical origin of the enhancement factors is essentially the nonperturbative contributions to the VCE which makes the VCE different from the original assumption (i), whereas the non- factorized part of the 4QC given in Ref. [6] is only a minor source of the enhancement factors. More precise nonperturbative QCD approach along this line is expected for developing practically useful algorithm to improve the application of the QCD sum rule to low energy processes.

\section{Acknowledgement}

This work is supported by the National Natural Science Foundation of China, the Fundamental Research Foundation of Tsinghua University, and a special grant from the State Commission of Education of China. We would like to thank K. Yamawaki and R.A. Bertlmann for interesting discussions. 


\section{REFERENCES}

[1] M.A. Shifman, A.L. Vainshtein, and V.I. Zakharov, Nucl. Phys. B147, 385 (1979).

[2] G. Launer, S. Narison, and R. Tarrach, Z. Phys. C26, 433 (1984).

[3] See for example H.G. Dosch, Univ.Heidelberg preprint HD-THEP-85-13; Y. Chung, H.G. Dosch, M. Kremer, and D. Schall, Z. Phys. C25, 151 (1984).

[4] M. Kremer, N.A. Papadopoulos, and K. Schilcher, Phys. Lett. 143B, 476 (1984).

[5] R.A. Bertlmann, C.A. Dominguez, M. Loewe, M. Perrottet, and E. de Rafael, Z. Phys. C39, 231 (1988).

[6] Q. Wang, Y.-P. Kuang, Y.-P. Yi, and T.-F. Cai, Phys. Lett. B336, 70 (1994).

[7] J. Bijnens, C. Bruno, and E. de Rafael, Nucl. Phys. B390, 501 (1993).

[8] H.-J. He and Y.-P. Kuang, Z. Phys. C47, 565 (1990).

[9] K. Yamawaki and V.I. Zakharov, Preprint UM-TH-94-20, DPNU-94-23 (hepph/9406399).

[10] A. Manohar and H. Georgi, Nucl. Phys. B234, 232 (1984).

[11] S. Weinberg, Phys. Rev. Lett., 65, 1181 (1990).

[12] Particle Data Group, Phys. Rev. D54, 77 (1997). 